\title{
ON APPROXIMATION BY BOUNDED ANALYTIC FUNCTIONS $\left({ }^{1}\right)$
}

\author{
BY \\ J. L. WALSH
}

Introduction. Although this topic has been treated in a number of papers $[2 ; 5 ; 6 ; 9 ; 13]$, important further progress is now possible thanks to a highly useful theorem on the conformal mapping of multiply connected regions [12]; this new theorem is used in combination with suitable refinements of older methods. Previous results have dealt primarily with rather rough (geometric) degrees of convergence and their relations to regions of convergence and regions of analyticity of functions approximated. The new theorem on conformal mapping combined with new results [13] on series of interpolation of rational functions enables us to treat here more refined degrees of convergence and their relations to regions of analyticity and to smoothness (e.g., Lipschitz conditions) on the boundary, of functions approximated. We consider ( $\$ \$ 1-6)$ degree of approximation on the common boundary of a region in which the approximating functions are analytic and of a smaller region in which the approximated function is analytic, a problem which is relatively new [13], and consider also ( $\$ 7)$ measure of approximation on a suitable curve or set of curves interior to a given region in which the functions are analytic, an older problem [2].

A function $f(z)$ analytic in a one-sided neighborhood of a Jordan curve $C$ and continuous on $C$ is said to be of class $L(p, \alpha)$ on $C$, where $p(\geqq 0)$ is integral and $0<\alpha<1$, if $f(z)$ has a one-dimensional pth derivative on $C$ which satisfies there a Lipschitz condition of order $\alpha$. If $C$ is analytic or even fulfills merely mild geometric conditions, then $f^{(p)}(z)$ exists [7, Theorem 2.2] also as a twodimensional derivative. Of course $f^{(0)}(z) \equiv f(z)$.

The definition just given has no meaning if $p<0$; for such integral values of $p, 0<\alpha \leqq 1$, if $C$ is an analytic Jordan curve, we say that $f(z)$ is of class $L(p, \alpha)$ on $C$ provided $f(z)$ is analytic in a one-sided neighborhood of $C$ and $C$ can be expressed as the level locus $u(z)=1$ of a nonconstant function $u(z)$ harmonic in an annulus containing $C$ and having no critical point in the annulus, and where in the neighborhood of $C$ we have $|f(z)| \leqq M(1-\rho)^{p+\alpha}$ on the locus $u(z)=\rho, \cdot \rho_{0}<\rho<1, \rho_{0}<1$; here $M$ is to be independent of $z$ and $\rho$. To be sure, this requirement is a restriction on the behavior of $f(z)$ not on $C$ but in a one-sided neighborhood of $C$. Nevertheless, as Hardy and Littlewood have shown, if $C$ is the unit circle, and as also is true for $C$ an arbitrary

Received by the editors October 8, 1956.

${ }^{1}$ This research was sponsored (in part) by the U. S. Air Force, Office of Scientific Research of the Air Research and Development Command. 
analytic Jordan curve $[4, \S 5.2]$, whenever $f(z)$ is of class $L(p, \alpha)$ on $C$, $0<\alpha<1$, the derivative and integral if single valued of $f(z)$ are of respective classes $L(p-1, \alpha)$ and $L(p+1, \alpha)$ on $C$ when suitably defined on $C$ (if necessary); so even in the case $p<0$ the class $L(p, \alpha)$ is closely related to behavior on $C$. The classes $L(p, \alpha)$ are invariant under one-to-one conformal mapping of a region containing $C$. [Compare $8, \S 5$ ].

1. Theorem 1, direct. Our main theorem is

THEOREM 1. Let $D$ be a finite region of the z-plane whose boundary consists of mutually disjoint Jordan curves $B_{1}, B_{2}, \cdots, B_{\mu}, C_{1}, C_{2}, \cdots, C_{\nu}$, and let $U(z)$ be the function harmonic in $D$, continuous in the closure of $D$, and equal to zero and unity on $B=\sum B_{j}$ and $C=\sum C_{j}$ respectively. For every $\sigma, 0<\sigma<1$, let $\Gamma_{\sigma}$ denote the locus $U(z)=\sigma$ in $D$, and let $D_{\sigma}$ denote the subregion $0<U(z)<\sigma$ of $D$, whose boundary is $B+\Gamma_{\sigma}$.

If $\Gamma_{\rho}$ has no multiple point, and if the function $f(z)$ is analytic in $D_{\rho}$, continuous on $B$, and of class $L(p, \alpha)$ on $\Gamma_{\rho}, 0<\alpha<1$, then there exist functions $f_{n}(z)$ analytic in $D$ and continuous on $B$ such that $(n=1,2,3, \cdots)$

$$
\begin{aligned}
\left|f(z)-f_{n}(z)\right| & \leqq A_{1} e^{-n \rho / \tau} / n^{p+\alpha}, z \text { on } B, \\
\left|f_{n}(z)\right| & \leqq A_{2} e^{n(1-p) / \tau} / n^{p+\alpha}, z \text { in } D,
\end{aligned}
$$

where $2 \pi \tau$ is the total variation along $\Gamma_{\rho}$ of the function conjugate to $U(z)$.

Reciprocally, if $f(z)$ is defined on $B$, if the functions $f_{n}(z)$ are continuous on $B$ and analytic in $D$, and if (1) and (2) are valid for some integer $p$ and $0<\alpha<1$, then $f(z)$ can be defined so as to be analytic in $D_{\rho}$, continuous on $B$, and of class $L(p-1, \alpha)$ on $\Gamma_{\rho}$.

Here and below letters $A$ with or without subscripts represent constants, which can change from one formula to another, but which are independent of $n$ and $z$.

The region $D$ can be mapped [12] on a region $D^{\prime}: e^{d_{0}}<|u(z)|<e^{d_{1}}$,

$$
\begin{aligned}
u(z) \equiv \frac{\left(z-a_{1}\right)^{m_{1}}\left(z-a_{2}\right)^{m_{2}} \cdots\left(z-a_{\mu}\right)^{m_{\mu}}}{\left(z-b_{1}\right)^{n_{1}}\left(z-b_{2}\right)^{n_{2}} \cdots\left(z-b_{\nu}\right)^{n_{\nu}}}, \\
m_{j}>0, n_{j}>0, \sum m_{j}=\sum n_{j}=1,
\end{aligned}
$$

where the $a_{j}$ and $b_{j}$ are finite and are separated from $D^{\prime}$ by the images of the $B_{j}$ and $C_{j}$ respectively. Thus it is no loss of generality to prove Theorem 1 , as we do, for the case that $D$ is the region $D^{\prime}$. We suppose $D=D^{\prime}$ to lie interior to $C_{1}$, and we use a series of interpolation designed specifically for this specialized region $D$, namely the series $[13$, Theorem 1$]$

(t) $\quad f_{1}(z) \equiv \sum_{0}^{\infty} c_{n} u_{n}(z), \quad u_{0}(z) \equiv 1, \quad u_{n}(z) \equiv \frac{\left(z-\alpha_{1}\right) \cdots\left(z-\alpha_{n}\right)}{\left(z-\beta_{1}\right) \cdots\left(z-\beta_{n}\right)}$,

Where each $\alpha_{j}$ is some $a_{k}$ and each $\beta_{j}$ is some $b_{k}$, and we have 


$$
0<A_{3} \leqq\left|\frac{u_{n}(z)}{[u(z)]^{n}}\right| \leqq A_{4},
$$

uniformly for all $n$ and for all $z$ on any compact containing no $a_{j}$ or $b_{j}$.

For $z$ in $D_{\rho}$, the function $f(z)$ can be expressed

$$
\begin{gathered}
f(z) \equiv \phi_{1}(z)+\phi_{2}(z), \quad \phi_{1}(z)=\sum \phi_{k 1}(z), \quad \phi_{2}(z) \equiv \sum \phi_{k 2}(z), \\
\phi_{k 1}(z) \equiv \frac{1}{2 \pi i} \int_{\gamma_{k}} \frac{f(t) d t}{t-z}, \quad \phi_{k 2}(z) \equiv \frac{1}{2 \pi i} \int_{B_{k}} \frac{f(t) d t}{t-z},
\end{gathered}
$$

where $\gamma_{k}$ is a suitably chosen rectifiable Jordan curve in $D_{\rho}$ near $\Gamma_{\rho}$, depending on $z$, precisely one such curve near each component of $\Gamma_{\rho}, z$ interior to a region bounded by $B$ and the $\gamma_{k}$, and where the integrals are taken in the positive sense with respect to that region. The function $\phi_{1}(z)$ is analytic throughout $D_{0}^{\prime}:|u(z)|<e^{d_{0}+\rho\left(d_{1}-d_{0}\right)}$, and can be represented there by a series (4). Moreover $\phi_{2}(z)$ is analytic on $\Gamma_{\rho}$, so $\phi_{1}(z)$ is of class $L(p, \alpha)$ on $\Gamma_{\rho}$.

The poles $\beta_{j}$ of $u_{n}(z)$ are to be found among the $b_{k}$, so lie exterior to $D_{0}^{\prime}$. Suppose now $p \geqq 0$. If $s_{n}(z)$ is the sum of the first $n+1$ terms of the series in (4), we have for $z$ in $D_{0}^{\prime}$ the interpolation formula

$$
\begin{aligned}
\phi_{1}(z)-s_{n}(z) & \equiv \frac{1}{2 \pi i} \int_{\gamma} \frac{\omega_{n}(z) \phi_{1}(t) d t}{\omega_{n}(t)(t-z)}, \quad \gamma=\sum \gamma_{k}, \\
\omega_{n}(z) & \equiv\left(z-\alpha_{n+1}\right) u_{n}(z) ;
\end{aligned}
$$

here $s_{n}(z)$ is the unique rational function of degree $n$ whose poles lie in the set $\left(\beta_{1}, \beta_{2}, \cdots, \beta_{n}\right)$ and which interpolates to $\phi_{1}(z)$ in the points $\left(\alpha_{1}, \alpha_{2}, \cdots\right.$, $\left.\alpha_{n+1}\right)$. A particular case of (7) occurs if $\phi_{1}(z)$ is replaced by an arbitrary rational function $R_{n}(z)$ of degree $n$ all of whose poles lie in the set $\left(\beta_{1}, \beta_{2}, \cdots\right.$, $\left.\beta_{n}\right)$,

$$
0 \equiv \frac{1}{2 \pi i} \int_{\gamma} \frac{\omega_{n}(z) R_{n}(t) d t}{\omega_{n}(t)(t-z)}, z \text { in } D_{0}^{\prime} .
$$

We shall use a combination of (7) and (8):

$$
\phi_{1}(z)-s_{n}(z) \equiv \frac{1}{2 \pi i} \int_{\gamma} \frac{\omega_{n}(z)\left[\phi_{1}(t)-R_{n}(t)\right] d t}{\omega_{n}(t)(t-z)}, z \text { in } D_{0}^{\prime} .
$$

Under the present circumstances, that $\phi_{1}(z)$ is of class $L(p, \alpha)$ on $\Gamma_{\rho}$, it follows from a result due to J. H. Curtiss, W. E. Sewell, H. M. Elliott [11, $\S 2]$, and Walsh [14] that functions $R_{n}(z)$ exist such that $(n>0)$

$$
\left|\phi_{1}(z)-R_{n}(z)\right| \leqq A / n^{p+\alpha}, z \text { on } \Gamma_{\rho} .
$$

Moreover we may replace $\gamma$ in (9) by $\Gamma_{\rho}$. The inequality

$$
\left|\phi_{1}(z)-s_{n}(z)\right| \leqq A_{1} e^{-n \rho / \tau} / n^{p+\alpha}, z \text { on } B,
$$


now follows from (10), (9), (7), (5), and (3), when we note the relations

$$
U(z) \equiv \frac{\log |u(z)|-d_{0}}{d_{1}-d_{0}}, \quad \tau=\frac{1}{d_{1}-d_{0}} .
$$

We may now set

$$
s_{n+1}(z)-s_{n}(z) \equiv c_{n+1} u_{n+1}(z)
$$

whence from (11)

$$
\left|c_{n+1} u_{n+1}(z)\right| \leqq 2 A_{1} e^{-n \rho / \tau} / n^{p+\alpha}, z \text { on } B
$$

and by means of a lemma [13, Lemma 3$]$ concerning the functions $u_{n}(z)$, for all $n(>0)$

$$
\begin{aligned}
\left|c_{n+1} u_{n+1}(z)\right| & \leqq A_{2} e^{n(1-\rho) / \tau} / n^{p+\alpha}, z \text { on } C, \\
\left|s_{n+1}(z)\right| & \leqq A_{3} e^{n(1-\rho) / \tau} / n^{p+\alpha}, z \text { on } C ;
\end{aligned}
$$

this last step will be discussed in more detail below. Since $f(z)$ is continuous on $B$ and $\phi_{1}(z)$ is analytic there, $\phi_{2}(z) \equiv f(z)-\phi_{1}(z)$ is continuous on $B$ and is analytic in $D+C$, so we have (1) and (2) from (11) and (13) by setting $f_{n}(z) \equiv s_{n}(z)+\phi_{2}(z)$.

It remains to justify the derivation of (13) from (12), which we do by remarking that for $\rho_{1}>1, p+\alpha>0$, we have

$$
\sum_{1}^{m} \frac{\rho_{1}^{n}}{n^{p+\alpha}} \leqq A \frac{\rho_{1}^{m}}{m^{p+\alpha}},
$$

where $A$ is independent of $m$. It is sufficient to take $m$ even, and we write

$$
\begin{aligned}
\sum_{1}^{m / 2} \frac{\rho_{1}^{n}}{n^{p+\alpha}} & \leqq \sum_{1}^{m / 2} \rho_{1}^{n} \leqq A^{\prime} \rho_{1}^{m / 2}, \\
\sum_{(m / 2)+1}^{m} \frac{\rho_{1}}{n^{p+\alpha}} & \leqq \frac{1}{(m / 2+1)^{p+\alpha}} \sum_{(m / 2)+1}^{m} \rho_{1}^{n} \leqq A^{\prime \prime} \frac{\rho_{1}^{m}}{m^{p+\alpha}},
\end{aligned}
$$

so (14) follows, as does the first part of Theorem 1 for $p \geqq 0$.

Incidentally, we remark that (12) and (5) yield

$$
\left|c_{n}\right| \leqq A e^{\left[(\rho-1) d_{0}-\rho d_{1}\right] n} / n^{p+\alpha} .
$$

If $f(z)$ is of class $L(p, \alpha), p<0$, we use (7) with the choice of $\Gamma_{r}$ for $\gamma$, $r=r_{n}=\rho(1-1 / n)$. For $z$ on $B$ we have by (5) with $n>0$

$$
\begin{aligned}
& \left|\phi_{1}(z)-s_{n}(z)\right| \leqq A e^{n d_{0}}(\rho / n)^{p+\alpha} / e^{n\left[d_{0}+\rho(1-1 / n) / \tau\right]}, \\
& \left|\phi_{1}(z)-s_{n}(z)\right| \leqq A_{1} e^{-n \rho / \tau} / n^{p+\alpha},
\end{aligned}
$$

which in form is identical with (11).

To operate conveniently with (16) we need the remark that for $\rho_{1}>1$, 
$p+\alpha<0$, we have

$$
\sum_{n=1}^{m} \frac{\rho_{1}^{n}}{n^{p+\alpha}} \leqq A \frac{\rho_{1}^{m}}{m^{p+\alpha}},
$$

where $A$ is independent of $m$. We need merely write

$$
\sum_{n=1}^{m} \frac{\rho_{1}^{n}}{n^{p+\alpha}} \leqq \frac{1}{m^{p+\alpha}} \sum_{n=1}^{m}{ }_{\rho_{1}}^{n},
$$

from which (17) follows.

We now set $s_{n+1}(z)-s_{n}(z) \equiv c_{n+1} u_{n+1}(z)$, whence by (16) for $n$ sufficiently large

$$
\left|c_{n+1} u_{n+1}(z)\right| \leqq 2 A_{1} e^{-n \rho / \tau} / n^{p+\alpha}, z \text { on } B
$$

so (12) follows [13, Lemma 3$]$ for all $n(>0)$, and (13) is a consequence of (17). As before, the function $\phi_{2}(z) \equiv f(z)-\phi_{1}(z)$ is continuous on $B$ and is analytic in $D+C$, so (1) and (2) are deduced from (16) and (13) by setting $f_{n}(z) \equiv s_{n}(z)+\phi_{2}(z)$. Incidentally, (15) also follows.

2. Theorem 1, indirect. We have now completed the proof of the first part of Theorem 1 , and proceed to prove the second part. With $p \geqq 1,0<\alpha<1$, and with (1) and (2) as hypothesis, we write for $n$ sufficiently large

$$
\begin{aligned}
& \left|f_{n+1}(z)-f_{n}(z)\right| \leqq A_{3} e^{-n \rho / \tau} / n^{p+\alpha}, z \text { on } B, \\
& \left|f_{n+1}(z)-f_{n}(z)\right| \leqq A_{4} e^{n(1-\rho) / \tau} / n^{p+\alpha}, z \text { on } C .
\end{aligned}
$$

Equation (18) requires no special interpretation, for the $f_{n}(z)$ are continuous on $B$, but (19) may be valid merely in the sense of (Fatou) limiting values, which exist on $C$ for almost all values of the conjugate of $U(z)$. The twoconstant theorem, a generalization of Hadamard's three circle theorem, relating the modulus of $f_{n+1}(z)-f_{n}(z)$ on $B, \Gamma_{\rho}$, and $C$, can be written

$$
\left|\begin{array}{ccc}
\log A_{3}-n \rho / \tau-(p+\alpha) \log n & 0 & 1 \\
\log M_{n} & \rho & 1 \\
\log A_{4}+n(1-\rho) / \tau-(p+\alpha) \log n & 1 & 1
\end{array}\right| \leqq 0
$$

where $M_{n}=\left[\max \left|f_{n+1}(z)-f_{n}(z)\right|, z\right.$ on $\left.\Gamma_{\rho}\right]$. Subtraction of the first row from the third row yields

$$
M_{n} \leqq A_{5} / n^{p+\alpha} .
$$

The sequence $f_{n}(z)$ converges uniformly throughout the closure of $D_{\rho}$, necessarily by (1) to a function analytic in $D_{\rho}$ and coinciding with $f(z)$ on $B$, because we have for $z$ on $\Gamma_{\rho}$

$$
\begin{aligned}
\left|f(z)-f_{n}(z)\right| & \leqq\left|f_{n+1}(z)-f_{n}(z)\right|+\left|f_{n+2}(z)-f_{n+1}(z)\right|+\cdots \\
& \leqq M_{n}+M_{n+1}+\cdots \leqq A_{6} / n^{p+\alpha-1}
\end{aligned}
$$


It follows from a theorem due to the present writer [9, Theorem 1], by virtue also of (2), that $f(z)$ belongs to $L(p-1, \alpha)$ on $\Gamma_{\rho}$.

If $p<1$, we again use the two-constant theorem to determine a bound for $M_{n}(r)=\left[\max \left|f_{n+1}(z)-f_{n}(z)\right|, z\right.$ on $\left.\Gamma_{r}\right], 0<r<\rho$, by means of (18) and (19). We obtain

$$
M_{n}(r) \leqq A_{7} e^{-n(\rho-r) / \tau} / n^{p+\alpha},
$$

which is indeed valid for all values of $p$, where $A_{\boldsymbol{z}}$ is independent of $n$ and $r$ For $z$ on $\Gamma_{r}$ we have

$$
\begin{aligned}
|f(z)| & \leqq A_{8} \sum_{m=2}^{\infty} e^{-m(\rho-r) / \tau} / m^{p+\alpha} \leqq A_{9} \int_{0}^{\infty} e^{-(\rho-r) x / \tau} x^{-p-\alpha} d x \\
& =A_{9} \Gamma(1-p-\alpha)\left(\frac{\tau}{\rho-r}\right)^{1-p-\alpha} \leqq A_{9}^{\prime}(\rho-r)^{p+\alpha-1},
\end{aligned}
$$

where $A_{9}^{\prime}$ is independent of $r$, so $f(z)$ is $L(p-1, \alpha)$ on $\Gamma_{\rho}$, and Theorem 1 is established.

In the second part of Theorem 1, we may replace (1) and (2) as hypothesis by (18) and (19), and define $f(z)$ on $B$ as the limit of the convergent sequence $f_{n}(z)$.

In the first part of Theorem 1 we have established (1) and (2) where $2 \pi \tau$ denotes the total variation of the conjugate of $U(z)$ along $\Gamma_{p}$; this is of course more specific than leaving $\tau$ unrestricted. But in the second part of Theorem 1 , the number $\tau(>0)$ is entirely arbitrary; compare $\$ 8$ below.

3 . Convergence on other level loci. We add several complements to Theorem 1. From (1) and (2) we deduce (18) and (19) and hence (20), for all $p$ and for all $r, 0 \leqq r \leqq 1$. For $p+\alpha>0$ and $r<\rho$ we obviously have

$$
\sum_{n=m}^{\infty} e^{-n(\rho-r) / \tau} / n^{p+\alpha} \leqq m^{-p-\alpha} \sum_{n=m}^{\infty} e^{-n(\rho-r) / \tau} \leqq A e^{-m(\rho-r) / \tau} / m^{p+\alpha} ;
$$

for $p+\alpha<0$ and $r<\rho$ this same inequality between the two extremes results through repeated integration by parts of the integral $(0<s<1, \beta>1)$

$$
\begin{aligned}
\int_{\beta}^{\infty} s^{x} x^{-p-\alpha} d x= & \frac{1}{(-\log s)^{-p-\alpha+1}} \int_{-\beta \log s}^{\infty} e^{-y 1} y^{-p-\alpha} d y \\
= & \frac{1}{(-\log s)^{-p-\alpha+1}}\left[s^{\beta}(-\log s)^{-p-\alpha} \beta^{-p \alpha \alpha}\right. \\
& \left.-(p+\alpha) \int_{-\beta \log s}^{\infty} e^{-y} y^{-p-\alpha-1} d y\right]
\end{aligned}
$$

whose modulus is then not greater than $A^{\prime}{ }_{S^{\beta}} \beta^{-p-\alpha}$, where $s$ is constant and $A^{\prime}$ is independent of $\beta$. Thus we estimate $\left|f(z)-f_{n}(z)\right|$ on $\Gamma_{r}$. For $r>\rho$ we use (14) and (17) with (20) to estimate $\left|f_{n}(z)\right|$ on $\Gamma_{r}$. There results 
COROllaRy 1. Under the conditions of either the first or second part of Theorem 1 we have

$$
\begin{aligned}
\left|f(z)-f_{n}(z)\right| & \leqq A_{3} e^{-n(\rho-r) / \tau} / n^{p+\alpha}, z \text { on } \Gamma_{r}, & & 0 \leqq r<\rho, \\
\left|f_{n}(z)\right| & \leqq A_{4} e^{n(r-\rho) / \tau} / n^{p+\alpha}, \text { z on } \Gamma_{r}, & & \rho<r \leqq 1 .
\end{aligned}
$$

Although Theorem 1 does not admit the possibility $p+\alpha=0$, Corollary 1 is valid as a consequence of (1) and (2) even if $p+\alpha=0$.

In the proof of (1) and (2) for $p>0$, it is necessary for the reasoning as given that $\Gamma_{\rho}$ have no multiple point, because otherwise (10) has not been established; but this restriction on $\Gamma_{\rho}$ is not necessary for the other parts of Theorem 1. To be sure, the classes $L(p, \alpha)$ on $\Gamma_{\rho}, p<0$, have not been formally defined for the case that $\Gamma_{\rho}$ has multiple points; but the definition extends in a natural and obvious way, in terms of the level loci of the function $U(z)$. The discrepancy of unity in the exponents of $n$ between the direct and indirect parts of Theorem 1 is inherent in the nature of the problem $[4, \S 6.4$; compare also $\$ 4$ below].

4. Other classes of functions. Theorem 1 intentionally omits consideration of the class $L(p, \alpha)$ for $\alpha=1$. A suitable new class with properties analogous to those of $L(p, \alpha)$ for $p \geqq 0,0<\alpha<1$, was introduced into the study of degree of trigonometric approximation by Zygmund, a class whose present analogue we denote by $Z_{p}$. If $C$ is a rectifiable Jordan curve, and if $f_{1}(s) \equiv f(z)$ rerepresents the boundary values on $C$ of a function $f(z)$ analytic in a onesided neighborhood of $C$ and continuous on $C$, where $s$ is arc length on $C$, then $f(z)$ is said to belong to $Z_{p}(p \geqq 0)$ on $C$ if $f_{1}^{(p)}(s)$ exists and is continuous on $C$, and there satisfies the condition

$$
\left|f_{1}^{(p)}(s+h)+f_{1}^{(p)}(s-h)-2 f_{1}^{(p)}(s)\right| \leqq A|h|,
$$

where $A$ is independent of $h$ and $s$. If the curve $C$ is analytic, then that $f(z)$ belong to $Z_{p}$ is both necessary and sufficient for the existence of a sequence of polynomials in $z$ of respective degrees $n$ converging to $f(z)$ on $C$ with degree of convergence $A_{1} / n^{p+1}$ [see $8, \S 4$ ].

In defining a sequence of new classes of analytic functions on an analytic Jordan curve $C$, we take $Z_{-2}=L(-2,1)$, as defined in the Introduction, to be fundamental; the class $Z_{p-2}, p>0$, is the class of $p$ th iterated integrals of functions of class $Z_{-2}$; the class $Z_{p-2}, p<0$, is the class of $p$ th derivatives of functions of class $Z_{-2}$. Thus we have a sequence of classes $\cdots, Z_{-2}, Z_{-1}$, $Z_{0}, Z_{1}, \cdots$, where the derivative and integral of a function of any class belong respectively to the next lower and next higher class. It is then true $[8, \S 5]$ that the class $Z_{p-2}, p<0$, is precisely the class $L(p-2,1)$, and the class $Z_{p-2}$ for $p \geqq 2$ is identical with the class $Z_{p-2}$ previously defined.

The methods used above apply if $p \neq-1$ to the study of these new classes $Z_{p}$ of functions, so we have 
Corollary 2. With the hypothesis and notation of Theorem 1 on $D, B_{j}, C_{j}$, $B, C, U(z), \Gamma_{\rho}$, and $D_{\rho}$, the conclusions of Theorem 1 persist if $L(p, \alpha)$ and $L(p-1, \alpha)$ are replaced by $Z_{p}$ and $Z_{p-1}$ respectively and if we set $\alpha=1$ in (1) and (2), provided $p \neq-1$ in the first part of Theorem 1 and $p \neq 0$ in the second part.

The inequality (15) follows in the nonexceptional case $p \neq-1$ of the first part of Corollary 2.

The case $p=-1$ is exceptional in the treatment already given of the first part of Theorem 1. Indeed, a function of class $Z_{-1}$ on a Jordan curve $C$ is not defined directly in terms of its own properties on $C$, but rather as the integral of a function of class $Z_{-2}$ or the derivative of a function of class $Z_{0}$, so the preceding methods do not apply. The class $Z_{-1}$ itself is unusual in its properties. The function $f(z) \equiv 1 /(1-z)$ is of class $Z_{-2}=L(-2,1)$ on $\gamma:|z|=1$, so its indefinite integral $-\log (1-z)$ is by definition of class $Z_{-1}$, yet is obviously not of class $L(-1,1)$ on $\gamma$; that is to say, the indefinite integral of $f(z)$ is not bounded in the neighborhood of $\gamma$. Likewise the case $p=0, \alpha=1$ is exceptional in the treatment already given of the second part of Theorem 1 . Suppose (1) and (2) are given with $p=0, \alpha=1$. We deduce (20) with $p+\alpha=1$, but $(21)$ for $z$ on $\Gamma_{r}(0<r<\rho)$ is replaced by

$$
|f(z)| \leqq A_{8} \sum_{\nu=1}^{\infty} e^{-\nu(\rho-r) / \tau} / \nu \leqq-A_{9} \log \left(1-e^{-(\rho-r) / \tau}\right) \leqq A_{9}^{\prime} \log (\rho-r),
$$

so this method does not establish the boundedness of $|f(z)|$ in $D_{\rho}$; such boundedness is the analogue of the requirement on the modulus of $f(z)$ for $p<-1$. Indeed, inequalities (1) and (2) with $p+\alpha=1, \mathrm{p}=0$, do not imply the boundedness of $f(z)$ in $D_{\rho}$, as is shown by the counterexample $f(z) \equiv \log (1-z)$, $B:|z|=1 / 2, C:|z|=2, f_{n}(z) \equiv-\sum_{1}^{n} z^{m} / m$, whence for $z$ on $B$

$$
\left|f(z)-f_{n}(z)\right| \leqq \sum_{n+1}^{\infty}|z|^{m} / m \leqq(1 / n) \sum_{n+1}^{\infty} 1 / 2^{m}=1 / 2^{n} \cdot n,
$$

and by (14) for $z$ on $C$

$$
\left|f_{n}(z)\right| \leqq \sum_{1}^{n} 2^{m} / m \leqq A 2^{n} / n .
$$

These inequalities are precisely of form (1) and (2), for we have

$$
U(z) \equiv \log (2|z|) /(2 \log 2), \quad \rho=1 / 2, \quad \tau=1 /(2 \log 2) .
$$

We later (Theorem 3 ) remove the restrictions $p \neq-1$ and $p \neq 0$ of Corollary 2.

5. Differentiation of sequences. In order to discuss the exceptional cases of Corollary 2, and also for its own intrinsic interest, we prove

Theorem 2. In the first part of Theorem 1, or Corollary 2, where D (interior 
to $C_{1}$ ) is the region $e^{d_{0}}<|u(z)|<e^{d_{1}}$ and $u(z)$ is given by (3), with $\phi_{1}(z)$ analytic throughout the region $|u(z)|<e^{d_{0}+\rho / \tau}$ and of class $L(p, \alpha)$ or $Z_{p}$ on the boundary, then whether $p$ is positive, negative (but $p \neq-1$ for $Z_{p}$ ) or zero we have

$$
\begin{aligned}
\left|\phi_{1}^{\prime}(z)-s_{n}^{\prime}(z)\right| & \leqq A_{1} e^{-n \rho / \tau} / n^{p+\alpha-1}, \text { z on } B, \\
\left|s_{n}^{\prime}(z)\right| & \leqq A_{2} e^{n(1-\rho) / \tau} / n^{p+\alpha-1}, \text { z in } D, \\
s_{n}(z) & \equiv \sum_{0}^{n} c_{k} u_{k}(z),
\end{aligned}
$$

where $s_{n}(z)$ is the partial sum of the formal expansion of type (4) of $\phi_{1}(z)$, and $\alpha=1$ for $Z_{p}$.

It is convenient first to establish a lemma (new notation).

Lemma 1. If the function $u(z)$ is analytic and has no critical point on the locus $C_{\lambda}:|u(z)|=\lambda$, and if the function $u_{n}(z)$ is analytic in the neighborhood of $C_{\lambda}$ and on every locus $C_{\eta}:|u(z)|=\eta$ satisfies the inequalities $\left(\lambda^{\prime \prime}<\lambda<\lambda^{\prime}\right)$

$$
\begin{array}{ll}
\left|u_{n}(z)\right| \leqq A \eta^{n}, \text { z on } C_{\eta}, & \lambda \leqq \eta \leqq \lambda^{\prime}, \\
\left|u_{n}(z)\right| \leqq A \lambda^{n}, \text { z on } C_{\eta}, & \lambda^{\prime \prime} \leqq \eta \leqq \lambda,
\end{array}
$$

for suitably chosen $\lambda^{\prime}$ and $\lambda^{\prime \prime}$, where $A$ is independent of $\eta$, then we have on $C_{\lambda}$

$$
\left|u_{n}^{\prime}(z)\right| \leqq A^{\prime} n \lambda^{n} \text {. }
$$

We use a method of proof which has already been employed by Sewell [ 4 , p. 38] for the case of polynomials. If $z_{0}$ is a point of $C_{\lambda}$, the distance $\delta$ from $z_{0}$ to the nearest point of $C_{\lambda+\lambda / n}$ is not less than $k \lambda / n$, for $n$ sufficiently large, where $k(>0)$ is suitably chosen independent of $z_{0}$. For $n$ sufficiently large we write

$$
u_{n}^{\prime}\left(z_{0}\right) \equiv \frac{1}{2 \pi i} \int_{\left|z-z_{0}\right|=\delta} \frac{u_{n}(z) d z}{\left(z-z_{0}\right)^{2}},
$$

whence by (25) and (26)

$$
\left|u_{n}^{\prime}\left(z_{0}\right)\right| \leqq A(\lambda+\lambda / n)^{n} / \delta \leqq A \lambda^{n}(1+1 / n)^{n} /(k \lambda / n),
$$

which implies $(27)\left({ }^{2}\right)$.

Under the hypothesis of Theorem 2, inequalities (15) and (27) yield (23) and (24) by the further inequalities used in proving Corollary 1 . Both Lemma 1 and Theorem 2 clearly extend to differentiation of higher order.

Theorem 2 is of significance in removing the restrictions of Corollary 2:

(2) For immediate application to the proof of Theorem 2, it is sufficient here to use the lemma: If $R_{n}(z)$ is a rational function of degree not greater than $n$ whose poles are in modulus greater than $R(>1)$, then the inequality $\left|R_{n}(z)\right| \leqq 1$ for $|z| \leqq 1$ implies $\left|R_{n}^{\prime}(z)\right| \leqq A n$ for $|z| \leqq 1$. This hypothesis implies $[1, \mathrm{p} .250]\left|R_{n}(z)\right| \leqq[(R Z-1) /(R-Z)]^{n}$ on $|z| \leqq Z, 1 \leqq Z<A$, so the hypothesis of Lemma 1 is fulfilled, with $u(z) \equiv z, \lambda=1$. 
THEOREM 3. Theorem 1 remains valid if $f(z)$ is given of class $Z_{p}$ on $\Gamma_{\rho}$, where we deduce (1) and (2) with $\alpha=1$, and if (1) and (2) are given with $\alpha=1$, where we conclude that $f(z)$ belongs to class $Z_{p-1}$ on $\Gamma_{\rho}$.

Thanks to Corollary 2 to Theorem 1 , we need treat here merely the respective cases $p=-1$ and $p=0$.

A rough indication of the proof of the first part of Theorem 3 is that we transform the $z$-plane onto the $z^{\prime}$-plane [as in 12], in which $D^{\prime}$ is the image of $D, D^{\prime}$ interior to the image of $C_{1}$, and we integrate the transform $F\left(z^{\prime}\right)$ of $f(z)$ (of class $Z_{-1}$ ), employ the development (4) for a suitable component of $F\left(z^{\prime}\right)$, and by differentiation complete the proof. However, the integral of $F\left(z^{\prime}\right)$ is not necessarily single-valued in $D_{\rho}^{\prime}$, the image of $D_{\rho}$, so we choose a suitable rational function

$$
r\left(z^{\prime}\right) \equiv \sum_{1}^{\mu} \frac{q_{k}}{z^{\prime}-a_{k}}+\sum_{2}^{\nu} \frac{q_{k}^{\prime}}{z^{\prime}-b_{k}}
$$

so that the indefinite integral $\Phi\left(z^{\prime}\right)$ of $F\left(z^{\prime}\right)+r\left(z^{\prime}\right)$ is single-valued in $D_{\rho}^{\prime}$. The given function $f(z)$ is by hypothesis of class $Z_{-1}$ on $\Gamma_{\rho}$, so $F\left(z^{\prime}\right)$ is of class $Z_{-1}$ on the image $\Gamma_{\rho}^{\prime}$ of $\Gamma_{\rho}$, and $\Phi\left(z^{\prime}\right)$ is of class $Z_{0}$ on $\Gamma_{\rho}^{\prime}$. Moreover, since $B^{\prime}$ is composed of a finite number of mutually disjoint analytic Jordan curves, $\Phi\left(z^{\prime}\right)$ has a continuous derivative on the image $B^{\prime}$ of $B$.

By the method used in (6), we express $\Phi\left(z^{\prime}\right)$ in $D^{\prime}$ as the sum $\Phi\left(z^{\prime}\right) \equiv \Phi_{1}\left(z^{\prime}\right)$ $+\Phi_{2}\left(z^{\prime}\right)$, where $\Phi_{1}\left(z^{\prime}\right)$ is analytic in $D_{\rho}^{\prime}$ and in the closed interiors of the curves composing $B^{\prime}$, and where $\Phi_{2}\left(z^{\prime}\right)$ is analytic exterior to the curves composing $B$. The development with partial sums $s_{n}\left(z^{\prime}\right)$ of $\Phi_{1}\left(z^{\prime}\right)$ similar to (4) is such that the analogue of (15) holds with $p=0, \alpha=1$, whence by Theorem 2

$$
\begin{aligned}
\left|\Phi_{1}^{\prime}\left(z^{\prime}\right)-s_{n}{ }^{\prime}\left(z^{\prime}\right)\right| & \leqq A_{1} e^{-n \rho / \tau}, \quad z \text { on } B^{\prime}, \\
\left|s_{n}^{\prime}\left(z^{\prime}\right)\right| & \leqq A_{2} e^{n(1-\rho) / \tau}, z \text { in } D^{\prime} .
\end{aligned}
$$

We now set $\Phi_{1}^{\prime}\left(z^{\prime}\right) \equiv \Phi^{\prime}\left(z^{\prime}\right)-\Phi_{2}^{\prime}\left(z^{\prime}\right) \equiv F\left(z^{\prime}\right)+r\left(z^{\prime}\right)-\Phi_{2}^{\prime}\left(z^{\prime}\right), \quad F_{n}\left(z^{\prime}\right) \equiv s_{n}^{\prime}\left(z^{\prime}\right)$ $-r\left(z^{\prime}\right)+\Phi_{2}^{\prime}\left(z^{\prime}\right)$, whence

$$
\begin{aligned}
\left|F\left(z^{\prime}\right)-F_{n}\left(z^{\prime}\right)\right| & \leqq A_{1} e^{-n \rho / \tau}, \quad z \text { on } B^{\prime}, \\
\left|F_{n}\left(z^{\prime}\right)\right| & \leqq A_{2} e^{n(1-\rho) / \tau}, z \text { in } D^{\prime} .
\end{aligned}
$$

The singularities of $F_{n}\left(z^{\prime}\right)$ on $B^{\prime}$ are precisely those of $F\left(z^{\prime}\right)$ on $B^{\prime}$, and $F\left(z^{\prime}\right)$ is continuous on $B^{\prime}$, so transformation to the $z$-plane now yields the first part of Theorem 3.

To prove the second part of Theorem 3, we note that the hypothesis (1) and (2) implies (18) and (19) with $p=0, \alpha=1$. The two-constant theorem then implies

$$
\begin{aligned}
& \left|f_{n+1}(z)-f_{n}(z)\right| \leqq A_{3} e^{-n(\rho-\epsilon) / \tau} / n, \quad z \text { on } \Gamma_{\epsilon}, \\
& \left|f_{n+1}(z)-f_{n}(z)\right| \leqq A_{4} e^{-n(\rho-1+\epsilon) / \tau} / n, z \text { on } \Gamma_{1-\epsilon},
\end{aligned}
$$


where $0<\epsilon<1-\rho, \epsilon<\rho$; the constants $A_{3}$ and $A_{4}$ vary continuously with $\epsilon$. The functions $u_{n}(z) \equiv f_{n+1}(z)-f_{n}(z)$ satisfy the conditions of Lemma 1 on both the curves $\Gamma_{\epsilon}$ and $\Gamma_{1-\epsilon}$, if we define $\Gamma_{\epsilon}$ by the equation $e^{[U(z)-\rho] / \tau}=e^{(\epsilon-\rho) / \tau}$ and (as is allowable) take $A$ in Lemma 1 as $A_{3} / n$, so we have

$$
\begin{aligned}
& \left|f_{n+1}^{\prime}(z)-f_{n}^{\prime}(z)\right| \leqq A_{5} e^{-n(\rho-\epsilon) / \tau}, \quad z \text { on } \Gamma_{\epsilon}, \\
& \left|f_{n+1}^{\prime}(z)-f_{n}^{\prime}(z)\right| \leqq A_{6} e^{-n(\rho-1+\epsilon) / \tau}, z \text { on } \Gamma_{1-\epsilon} .
\end{aligned}
$$

The methods already developed show that $f_{n}(z)$ converges to $f(z)$ throughout $D_{\rho}$, uniformly on any compact in $D_{\rho}$. It now follows by Corollary 2 to Theorem 1 that $f^{\prime}(z)$ is of class $Z_{-1}$ on $\Gamma_{\rho}$, so $f(z)$ is of class $Z_{0}$ on $\Gamma_{\rho}$, and Theorem 3 is established.

In connection with Theorem 1, of course we may differentiate in the original $z$-plane or in the auxiliary $z^{\prime}$-plane used in the proof of the first part of Theorem 3. A consequence of Lemma 1 and of Corollary 1 in extended form is

THEOREM 4. Under the hypothesis of the first or second part of Theorem 1 or of Theorem 3, but with $D$ interior to $C_{1}$, we have

$$
\begin{aligned}
\left|f^{\prime}(z)-f_{n}^{\prime}(z)\right| & \leqq A_{5} e^{-n(\rho-r) / \tau} / n^{p+\alpha-1}, \text { z on } \Gamma_{r}, & & 0<r<\rho, \\
\left|f_{n}^{\prime}(z)\right| & \leqq A_{6} e^{n(r-\rho) / \tau} / n^{p+\alpha-1}, \text { z on } \Gamma_{r}, & & \rho<r<1 .
\end{aligned}
$$

It is not to be expected that these inequalities should be valid also on $B$ and $C$ respectively, for the derivatives $f^{\prime}(z)$ and $f_{n}^{\prime}(z)$ need not exist on $B$ and $C$. But if the bounding curves $B_{j}$ and $C_{j}$ are analytic, and if $f(z)$ is analytic on $B$, these inequalities for $r=0$ and $r=1$ respectively follow from Theorem 2, where the $f_{n}(z)$ are the particular functions used in the proof of the first parts of Theorem 1 and Theorem 3.

6. Integration of sequences. The study of the integration of approximating sequences depends on a further lemma.

LEMMA 2. With the hypothesis of Lemma 1 on $u(z)$, suppose $u(z)$ has no critical point in the closed region $D_{1}$ bounded by $C_{\lambda}$ and $C_{\lambda^{\prime}}$, and suppose $(n>0)$

$$
\left|u_{n}(z)\right| \leqq A_{n}|u(z)|^{n}
$$

in $D_{1}$. Let $z$ be an arbitrary point of $D_{1}$, and let $z_{0}$ be the point on $C_{\lambda}$ on the level curve through $z$ of the function conjugate to $u(z)$. Set

$$
U_{n}(z) \equiv \int_{z_{0}}^{z} u_{n}(z) d z
$$

where the path of integration is the arc in $D_{1}$ of this level curve. Then we have

$$
\left|U_{n}(z)\right| \leqq A_{1} A_{n}|u(z)|^{n} /(n+1),
$$

where $A_{1}$ is independent of $n$ and of $z$. 
We write, by use of the fact that $u^{\prime}(z) \neq 0$ in the closure of $D_{1}$,

$$
\left|U_{n}(z)\right| \equiv\left|\int_{u\left(z_{0}\right)}^{u(z)} \frac{u_{n}(z)}{u^{\prime}(z)} d[u(z)]\right| \leqq \int_{\lambda}^{|u(z)|} \frac{\left|u_{n}(z)\right|}{\left|u^{\prime}(z)\right|} d[|u(z)|],|u(z)| \leqq \lambda^{\prime},
$$

where $1 /\left|u^{\prime}(z)\right|$ is bounded, whence the conclusion follows from (28).

Lemma 2 obviously has numerous applications in the situation of Theorem 1 , applications that are limited by the fact that $D$ is by definition multiply connected (even if $\mu=\nu=1$ ) and hence that indefinite integrals of the functions $f(z)$ and $f_{n}(z)$ need not be single-valued. For instance (20) and the inequalities of Corollary 1 are precisely of the form of (28). Instead of formulating rather complicated results to include broad classes of multiple valued integrals, we prove merely

THEOREM 5. Under the hypothesis of the first part of Theorem 1 or of Theorem 3 , with $D$ interior to $C_{1}$, suppose $\mu=\nu=1$, and suppose $f(z)$ and the $f_{n}(z)$ to be analytic throughout the closed interior of $B_{1}$. Let $z=a$ be an arbitrary point on or within $B_{1}$. Then we have

$$
\begin{array}{rlrl}
\left|\int_{a}^{z}\left[f(z)-f_{n}(z)\right] d z\right| & \leqq A_{5} e^{-n(\rho-r) / \tau} / n^{p+\alpha+1}, \text { z on } \Gamma_{r}, & & 0<r<\rho, \\
\left|\int_{a}^{z} f_{n}(z) d z\right| & \leqq A_{6} e^{n(r-\rho) / \tau} / n^{p+\alpha+1}, \text { z on } \Gamma_{r}, & \rho<r<1 .
\end{array}
$$

In connection with the hypothesis of Theorem 5, compare Theorem 6 below. The function $U(z)$ has no critical point in $D$ under the conditions of Theorem 5. These integrals may all be taken over a rectilinear path from $z=a$ to some fixed point $z_{1}$ on $\Gamma_{r^{\prime}}, 0<r^{\prime}<r$, then along $\Gamma_{r^{\prime}}$ to $z_{0}$ of Lemma 2, and along the level locus of the conjugate function of $U(z)$ to $z$. Along the first two parts of this path we have by Corollary 1 to Theorem 1 (extended to include $\alpha=1$ )

$$
\begin{aligned}
\left|f(z)-f_{n}(z)\right| & \leqq A_{3} e^{-n\left(p-r^{\prime}\right) / \tau} / n^{p+\alpha}, & & 0<r^{\prime}<r<\rho, \\
\left|f_{n}(z)\right| & \leqq A_{4} e^{n\left(r^{\prime}-\rho\right) / \tau} n^{p+\alpha}, & & \rho<r^{\prime}<r<1,
\end{aligned}
$$

so (29) and (30) follow from (1) and (2) by Corollary 1 and Lemma 2.

Theorem 5 applies in part under the conditions of Theorem 1 whenever $\Gamma_{\rho}$ consists of a single Jordan curve, $D$ interior to $C_{1}$, the $f(z)$ and $f_{n}(z)$ analytic throughout the closed interior of every $B_{j}$; for in that case $\Gamma_{r}$ also consists of a single Jordan curve when $r$ is sufficiently near $\rho$, and inequalities (29) and (30) are valid for such values of $r$. Theorem 5 does not extend without change to the general case $\nu>1$, for then the $f_{n}(z)$ may have multiple valued integrals. Theorem 5 does not extend without change to the case $\mu>1$, for it may be impossible to choose the indefinite integrals of all the $f_{n}(z)$ interior to $B_{1}$ as the analytic extensions of the indefinite integrals of the $f_{n}(z)$ interior to $B_{2}$, 
while preserving the inequalities of form (30); for an illustration of this fact compare $[1, \S 4.7]$.

Lemmas 1 and 2 apply with a hypothesis less precise than (1) and (2). If we require merely that $f(z)$ be analytic in $D_{\rho}$, we may replace (1) and (2) by [compare 2]

$$
\begin{aligned}
& \lim \sup \left[\max \left|f(z)-f_{n}(z)\right|, z \text { on } B\right]^{1 / n} \leqq e^{-\rho / \tau}, \\
& \left.\lim \sup \text { [l.u.b. }\left|f_{n}(z)\right|, z \rightarrow C\right]^{1 / n} \leqq e^{(1-\rho) / r} ;
\end{aligned}
$$

Lemma 1 yields at once by the analogue of Corollary 1

$$
\begin{aligned}
& \lim \sup \left[\max \left|f^{\prime}(z)-f_{n}^{\prime}(z)\right|, z \text { on } \Gamma_{r}\right]^{1 / n} \leqq e^{-(\rho-r) / \tau}, \quad 0<r<\rho, \\
& \lim \sup \left[\max \left|f_{n}^{\prime}(z)\right|, z \text { on } \Gamma_{r}\right]^{1 / n} \leqq e^{(r-\rho) / \tau}, \quad \rho<r<1 .
\end{aligned}
$$

These inequalities can also be obtained by differentiation from Cauchy's integral formula, with integration over $\Gamma_{r / 2}$ and $\Gamma_{r^{\prime}}, r^{\prime}>r, r^{\prime} \rightarrow r$. With the hypothesis of Theorem 5 except that $f(z)$ is required merely to be analytic throughout $D_{\rho}$, it follows by Lemma 2 that (29) and (30) may be replaced by

$$
\begin{aligned}
\limsup & {\left[\max \left|\int_{a}^{z}\left[f(z)-f_{n}(z)\right] d z\right|, z \text { on } \Gamma_{r}\right]^{1 / n} \leqq e^{-(\rho-r) / \tau}, } & 0<r<\rho, \\
& \limsup \left[\max \left|\int_{a}^{z} f_{n}(z) d z\right|, z \text { on } \Gamma_{r}\right]^{1 / n} \leqq e^{(r-\rho) / \tau}, & \rho<r<1 .
\end{aligned}
$$

These relations can also be obtained by elementary inequalities on the integrals.

7. Further analyticity requirements. We add a complement to Theorem 1 of somewhat different character:

Theorem 6. Suppose that the region $D$ of Theorem 1 lies interior to $C_{1}$, that the Jordan curves $B_{j}$ are analytic, and that in the first part of Theorem 1 or of Theorem 3 the function $f(z)$ is analytic on and within each $B_{j}$. Then the functions $f_{n}(z)$ in (1) and (2) can also be chosen analytic on and within each $B_{j}$.

We need to consider the map already used of the given region $D$ of Theorem 1 , together with the entities $B_{j}, C_{j}, B, C, U(z), \Gamma_{\sigma}, D_{\sigma}$, onto a region $D^{\prime}: e^{d_{0}}<\left|u^{\prime}\left(z^{\prime}\right)\right|<e^{d_{1}}$ of the $z^{\prime}$-plane where $u^{\prime}(z)$ is given by (3), where the other entities are denoted by $B_{j}^{\prime}, C_{j}^{\prime}, B^{\prime}, C^{\prime}, U^{\prime}\left(z^{\prime}\right), \Gamma_{\sigma}^{\prime}, D_{\sigma}^{\prime}$ respectively, and where the transform of $f(z)$ is $F\left(z^{\prime}\right)$. The map of $D$ onto $D^{\prime}$ is one-to-one and analytic not merely in those regions but if suitably extended, also in complete neighborhoods of $B$ and $B^{\prime}$, say throughout the closure of the region $D_{-\sigma}^{\prime}:-\sigma<U^{\prime}\left(z^{\prime}\right)<0, \sigma>0$. If $\sigma$ is suitably chosen, the functions $f_{n}(z)$ in (1) and (2) are analytic throughout the closure of $D_{-\sigma}$. Moreover it follows from the proof of Theorem 1 that for $z$ on $\Gamma_{-\sigma}$ we have [13, Equation (10)] for arbitrary $\delta(>0)$

$$
\left[\max \left|f(z)-f_{n}(z)\right|, z \text { on } \Gamma_{-\sigma}\right] \leqq A_{1} e^{-n(\sigma+\rho-\delta) / \tau} .
$$


We split $f(z)$ and the $f_{n}(z)$ into their components by integrating over a locus $\Gamma_{\mu}$, where $\Gamma_{\mu}$ is in $D$ near $\Gamma_{\rho}$, and integrating over $B$ or $\Gamma_{-\sigma}$ indifferently. For $z$ in $D_{\mu}$ we have $f_{n}(z) \equiv f_{n 1}(z)+f_{n 2}(z)$, with

$$
\begin{gathered}
f(z) \equiv \frac{1}{2 \pi i} \int_{\Gamma_{\mu}} \frac{f(t) d t}{t-z}, \quad f_{n 1}(z) \equiv \frac{1}{2 \pi i} \int_{\Gamma_{\mu}} \frac{f_{n}(t) d t}{t-z} \\
0 \equiv \frac{1}{2 \pi i} \int_{\Gamma_{-\sigma}} \frac{f(t) d t}{t-z}, \quad f_{n 2}(z) \equiv \frac{1}{2 \pi i} \int_{\Gamma_{-\sigma}} \frac{f_{n}(t) d t}{t-z}, \\
f_{n 2}(z) \equiv \frac{-1}{2 \pi i} \int_{\Gamma_{-\sigma}} \frac{\left[f(t)-f_{n}(t)\right] d t}{t-z} .
\end{gathered}
$$

By virtue of (31) we may now replace $f_{n}(z)$ in (1) and (2) by $f_{n 1}(z)$, with a suitable modification of $A_{1}$ and $A_{2}$ if necessary, which completes the proof of Theorem 6 .

The first studies $[2 ; 5]$ of approximation by bounded analytic functions were, like Theorem 6 , concerned with functions $f(z)$ and $f_{n}(z)$ analytic on and within each $B_{j}$; there the results were concerned primarily with geometric degree of convergence, as in the last part of $\S 6$.

Except for the case that $\Gamma_{\rho}$ has multiple points, Theorem 1 with Theorems 3 and 6 represents a relatively complete and satisfactory treatment of Problem $\beta$, the study of approximating functions bounded in a region $D_{0}=D+$ the closed interiors of the $B_{j}$, for approximation on a subset $D_{0}-D$ of $D_{0}$, where the function approximated is analytic on $D_{0}-D$ but not throughout $D_{0}$ and satisfies certain continuity conditions on a larger set $D_{0}-D+D_{\rho}+\Gamma_{\rho}$. This was formulated [6] in 1946 as an open problem which it would be desirable to solve.

By way of contrast, Problem $\alpha$ is concerned with the relation of continuity properties on $\Gamma_{\rho}$ of a function analytic on $D_{0}-D+D_{\rho}$, to degree of convergence on $\Gamma_{\rho}$ itself [compare 9 and 10].

No method is known for treating this Problem $\beta$ other than the one presented here, and indeed this Problem $\beta$ was the occasion for the preliminary investigation of the conformal mapping [12] used, and for the study of the special series of interpolation [13] whose properties are essential in our treatment. We have needed the relation

$$
0<A_{1} \leqq\left|\frac{\omega_{n}(z)}{[u(z)]^{n}}\right| \leqq A_{2},
$$

which itself is satisfied [compare 4, Theorem 2.5.7] if points of interpolation are chosen equally distributed on a level locus of $|u(z)|$, and the poles also equally distributed on such a level locus, the curves $C_{j}$ being analytic. But unless the development of $f(z)$ is a series of interpolation, such that the difference of two approximating functions $s_{n+1}(z)-s_{n}(z)$ is a rational function of degrce $n+1$, whose poles are known at least roughly in location, we are not 
in a position to derive (12) and (13) as a consequence of (11), and thus are not in a position to derive (2).

8. Extensions. Inequalities (1) and (2) are expressed for discrete values of $n=1,2,3, \cdots$. Functions $f_{\lambda}(z)$ exist for continuous values of $\lambda(\geqq 1)$ as well, if we set $f_{\lambda}(z) \equiv f_{n}(z)$ when $1 \leqq n \leqq \lambda<n+1$. The analogues of (1) and (2) persist:

$$
\begin{aligned}
&\left|f(z)-f_{\lambda}(z)\right| \leqq A_{1}^{\prime} e^{-\lambda \rho / \tau} / \lambda^{p+\alpha}, \quad z \text { on } B \\
&\left|f_{\lambda}(z)\right| \leqq A_{2}^{\prime} e^{\lambda(1-\rho) / \tau} / \lambda^{p+\alpha}, z \text { in } D .
\end{aligned}
$$

A change of variable here eliminates $\tau$, and we may still assume for the new $\lambda, \lambda \geqq 1$.

Theorems 1 and 3 have immediate applications to the study of extremal approximating functions. If $M(>0)$ is given, there exists [2] an extremal function $\phi_{M}(z)$ analytic and in modulus not greater than $M$ in $D$ such that $\left[\max \left|f(z)-\phi_{M}(z)\right|, z\right.$ on $\left.B\right]$ is least. Indeed, for fixed $M$ we consider a sequence of functions $\phi_{M}^{(j)}(z)$ each analytic and of modulus not greater than $M$ in $D$ such that the measure of approximation of $\phi_{M}^{(j)}(z)$ to $f(z)$ on $B$ approaches the greatest lower bound $m_{M}$ of measures for all such functions. In both parts of Theorem 1 (extended in the first part so that $f(z)$ is bounded but not necessarily continuous in a neighborhood of $B$ and in the second part so that $f_{n}(z)$ is not necessarily continuous on $B$ ) the function $f(z)$ exists analytic and bounded in some $D_{\sigma}(\sigma<\rho)$ taking almost everywhere on $B$ the given boundary values, so any limit of the sequence $\log \left|\phi_{M}^{(j)}(z)-f(z)\right|$ is dominated in $D_{\sigma}$ by a fixed function harmonic in $D_{\sigma}$ whose continuous boundary value taken on $B$ is $\log m_{M}$ and on $\Gamma_{\sigma}$ is some $\log M_{0}$ independent of $j$; thus an extremal $\phi_{M}(z)$ exists. Let $F_{n}(z)$ denote these extremal functions for the sequence

$$
M=A_{0} e^{n(1-\rho) / \tau} / n^{p+\alpha},
$$

where $A_{0}(>0)$ is arbitrary. For suitably chosen but fixed $k$ we have with the notation of (2) for every $n$ sufficiently large

$$
A_{2} e^{(n-k)(1-\rho) / \tau} /(n-k)^{p+\alpha} \leqq A_{0} e^{n(1-\rho) / \tau} / n^{p+\alpha},
$$

so it follows from Theorems 1 and 3 (slightly extended to admit discontinuities of $f(z)$ and the $f_{n}(z)$ on $B$ ) that we have for $n$ sufficiently large and with suitable choice of $A_{0}^{\prime}$

$$
\begin{gathered}
{\left[\max \left|f(z)-F_{n}(z)\right|, z \text { on } B\right] \leqq\left[\max \left|f(z)-f_{n-k}(z)\right|, z \text { on } B\right]} \\
\leqq A_{1} e^{-(n-k) \rho / \tau} /(n-k)^{p+\alpha} \leqq A_{0}^{\prime} e^{-n \rho / \tau} / n^{p+\alpha} ;
\end{gathered}
$$

with suitable choice of $A_{0}^{\prime}$ the inequality between these extreme members is valid for all $n$. Thus we have shown that under the hypothesis of Theorems 1 and 3 , if the $F_{n}(z)$ are the extremal functions corresponding to (32), then the extreme inequalities of (33) are valid. 
Again under the hypothesis of Theorems 1 and 3, if $m(>0)$ is given, there exists [2] an extremal function $\phi_{m}(z)$ analytic and of least maximum (or supremum) modulus in $D$ such that $\left[\max \left|f(z)-\phi_{m}(z)\right|, z\right.$ on $\left.B\right] \leqq m$. If $\Phi_{n}(z)$ is the sequence of extremal functions for the sequence $m=A e^{-n \rho / r} / n^{p+\alpha}$, where $A$ is arbitrary, it follows by the method just used that we have for every $n$

$$
\left|\Phi_{n}(z)\right| \leqq A^{\prime} e^{n(1-\rho) / \tau} / n^{p+\alpha}, z \text { in } D .
$$

Theorem 1 can be generalized by considering more general conditions than those of Lipschitz. If in the first part of Theorem 1 we replace the condition that $f(z)$ shall belong to $L(p, \alpha)$ by the condition that $f(z)$ be analytic in $D_{\rho}$, continuous on $\Gamma_{\rho}$, and that $f^{(p)}(z)$ should exist on $\Gamma_{\rho}$ and possess there the modulus of continuity $\omega(\delta)$, the second member of $(10)$ is replaced by $A \omega(1 / n) / n^{p}$, the analogue (and extension) of (14) is valid, and we have (1) and (2) except that $1 / n^{p+\alpha}$ is replaced by $\omega(1 / n) / n^{p}$ (compare [11, Theorem $2.1 ; 14])$. Conversely, suppose for $p \geqq 1$ that the $f_{n}(z)$ exist analytic in $D$ and continuous on $B$ such that

$$
\begin{aligned}
\left|f(z)-f_{n}(z)\right| & \leqq A_{1} e^{-n \rho / \tau} \Omega(n) / n^{p}, \quad z \text { on } B, \\
\left|f_{n}(z)\right| & \leqq A_{2} e^{n(1-\rho) / \tau} \Omega(n) / n^{p}, z \text { in } D,
\end{aligned}
$$

where $\Omega(x)$ is a non-negative function which is nonincreasing for $x$ sufficiently large such that $\int^{\infty}[\Omega(x) / x] d x$ exists. Then the two-constant theorem yields $M_{n} \leqq A_{5} \Omega(n) / n^{p}$ on $\Gamma_{\rho}$ by (34) and (35), and we have

$$
M_{n}+M_{n+1}+\cdots \leqq A_{6} \Omega(n) / n^{p-1},
$$

from which it follows [10, Theorem 1] that $f^{(p-1)}(z)$ exists and has a modulus of continuity $\omega(\delta)$ in terms of arc length on $\Gamma_{\rho}$ such that

$$
\omega(\delta) \leqq A_{7}\left[\delta \int_{a}^{a / \delta} \Omega(x) d x+\int_{1 / \delta}^{\infty}[\Omega(x) / x] d x\right], \quad 0<\delta \leqq 1 / a,
$$

where $a$ is suitably chosen independent of $\delta$.

9. Further remarks. Although results on the theory of approximation by polynomials [1] historically preceded results on approximation by bounded analytic functions, the latter can at times be used to yield simple proofs of known results [4] on approximation by polynomials. For instance let $B$ be a finite set of mutually exterior Jordan curves $B_{j}$, and suppose polynomials $f_{n}(z)$ of respective degrees $n(>0)$ are given such that

$$
\left|f(z)-f_{n}(z)\right| \leqq A_{1} e^{-n \delta} / n^{p+\alpha}, z \text { on } B,
$$

is valid $(0<\alpha \leqq 1, \delta>0)$. Then we have

$$
\left|f_{n+1}(z)-f_{n}(z)\right| \leqq A_{2} e^{-n \delta} / n^{p+\alpha}, z \text { on } B,
$$

and $f_{n+1}(z)-f_{n}(z)$ is a polynomial of degree $n+1$. Let $G(z)$ denote Green's function with pole at infinity for the infinite region $D^{\prime}$ bounded by $B$, and let 
$G_{\sigma}$ denote generically the locus $G(z)=\sigma(>0)$ in $D^{\prime}$. It follows by the generalized Bernstein Lemma $[1$, p. 77] from (37) that we have $(\gamma>\delta)$

$$
\left|f_{n+1}(z)-f_{n}(z)\right| \leqq A_{2} e^{n(\gamma-\delta)} / n^{p+\alpha}, z \text { on } G_{\gamma},
$$

from which we have by (14) and (17)

$$
\left|f_{n}(z)\right| \leqq A_{3} e^{n(\gamma-\delta)} / n^{p+\alpha}, z \text { on } G_{\gamma} .
$$

Inequalities (36) and (38) can be identified with (1) and (2), for in the notation of Theorem 1 , where $D$ is bounded by $B$ and $G_{\gamma}, U(z) \equiv G(z) / \gamma, \tau=1 / \gamma$, $\delta=\rho / \tau, \gamma-\delta=(1-\rho) / \tau$, whence $\Gamma_{\rho}$ is $G_{\delta}$, namely the locus $G(z)=\gamma \rho=\delta$. The conclusion of the second part of Theorem 3, a consequence merely of (36), is that $f(z)$ is of class $L(p-1, \alpha)$ or $z_{p-1}$ (if $\alpha=1$ ) on $G_{\delta}$ provided $G_{\delta}$ has no multiple points; this conclusion is independent of the auxiliary number $\gamma(>\delta)$.

It is in fact immaterial here whether the locus $G_{\gamma}$ consists of one or of several Jordan curves; in the latter case the second part of Theorem 1 applies to each region bounded by one of these Jordan curves and the subset of $B$ interior to it.

Theorem 1, thus applied to the study of approximation by polynomials, admits also a corresponding application to the study of approximation by more general rational functions, under suitable conditions. As an illustration, suppose $u(z)$ is defined by (3), and that for a function $f(z)$ defined merely on $B:|u(z)|=e^{d_{0}}$ we have $\left(d>d_{0}, 0<\alpha \leqq 1\right)$ for $n=1,2,3, \cdots$

$$
\left|f(z)-f_{n}(z)\right| \leqq A_{1} e^{-n\left(d-d_{0}\right)} / n^{p+\alpha}, z \text { on } B,
$$

where (in the notation of $\S 1$ ) $B$ is also $U(z)=0$, and $f_{n}(z)$ is a rational function of degree $n$ whose poles lie in the set $\left(\beta_{1}, \beta_{2}, \cdots, \beta_{n}\right)$; of course $f_{n}(z)$ need not be determined by interpolation, but may be for instance the rational function of the type prescribed of best approximation to $f(z)$ on $B$ in the sense of Tchebycheff with continuous norm function. From (39) we deduce for $n$ sufficiently large

$$
\left|f_{n+1}(z)-f_{n}(z)\right| \leqq 2 A_{1} e^{-n\left(d-d_{0}\right)} / n^{p+\alpha}, z \text { on } B .
$$

The function $f_{n+1}(z)-f_{n}(z)$ is a rational function of degree $n+1$ whose poles lie in the set $\left(\beta_{1}, \beta_{2}, \cdots, \beta_{n+1}\right)$, from which by (40) there follow [13, Lemma 3] with $C:|u(z)|=e^{d_{1}}, d_{1}>d$,

$$
\begin{aligned}
\left|f_{n+1}(z)-f_{n}(z)\right| & \leqq A_{3} e^{n\left(d_{1}-d\right)} / n^{p+\alpha}, z \text { on } C, \\
\left|f_{n}(z)\right| & \leqq A_{4} e^{n\left(d_{1}-d\right)} / n^{p+\alpha}, z \text { on } C .
\end{aligned}
$$

Inequalities (39) and (42) can be identified with (1) and (2), sufficient for the conclusion of the second part of Theorem 1 or of Theorem 3. It thus follows merely as a consequence of (39) that $f(z)$ can be extended from $B$ so as to be analytic interior to, and of class $L(p-1, \alpha)$ or $Z_{p-1}$ (if $\alpha=1$ ) on, the locus $|u(z)|=e^{d}$. 


\section{REFERENCES}

1. J. L. Walsh, Interpolation and approximation by rational functions in the complex domain, Amer. Math. Soc. Colloquium Publications, vol. 20, New York, 1935.

2. - On interpolation and approximation by functions analytic and bounded in a given region, Proc. Nat. Acad. Sci. U.S.A., vol. 24 (1938) pp. 477-486.

3. J. L. Walsh and W. E. Sewell, On the degree of polynomial approximation to analytic functions, Trans. Amer. Math. Soc. vol. 49 (1941) pp. 229-257.

4. W. E. Sewell, Degree of approximation by polynomials in the complex domain, Annals of Mathematics Studies, no. 9, Princeton, 1942.

5. E. N. Nilson and J. L. Walsh, Interpolation and approximation by functions analytic and bounded in a given region, Trans. Amer. Math. Soc. vol. 55 (1944) pp. 53-67.

6. J. L. Walsh, Taylor's series and approxination to analytic functions, Bull. Amer. Math. Soc. vol. 52 (1946) pp. 572-579.

7. J. L. Walsh, W. E. Sewell and H. M. Elliott, On the degree of polynomial approximation to harmonic and analytic functions, Trans. Amer. Math. Soc. vol. 67 (1949) pp. 381-420.

8. J. L. Walsh and H. M. Elliott, Polynomial approximation to harmonic and analytic functions: generalized continuity conditions. Trans. Amer. Math. Soc. vol. 68 (1950) pp. 183-203.

9. J. L. Walsh, Note on approximation by bounded analytic functions, Proc. Nat. Acad. Sci. U.S.A. vol. 37 (1951) pp. 821-826.

10. J. L. Walsh and H. M. Elliott, Degree of approximation on a Jordan curve, Proc. Nat. Acad. Sci. U.S.A. vol. 38 (1952) pp. 1058-1066.

11. H. M. Elliott, On approximation to analytic functions by rational functions, Proc. Amer. Math. Soc. vol. 4 (1953) pp. 161-167.

12. J. L. Walsh, On the conformal mapping of multiply connected regions, Trans. Amer. Math. Soc. vol. 82 (1956) pp. 128-146.

13. - Sur l'approximation par fonctions rationnelles et par fonctions holomorphes bornées, Annali di Matematica Pura ed Applicata (4) vol. 39 (1955) pp. 267-277.

14. - Note on degree of approximation to analytic functions by rational functions with preassigned poles, Proc. Nat. Acad. Sci. U.S.A. vol. 42 (1956) pp. 927-930.

HARVARD UNIVERSITY, Cambridge, Mass. 